\title{
Experiência da Atenção Integral à Saúde Individual e Familiar com enfoque na Responsabilização, Vínculo Médico-paciente, Ética e Profissionalismo no Currículo Médico Integrado
}

\author{
The Experience with Comprehensive Individual \\ and Family Healthcare with a Focus on \\ Accountability, the Physician-Patient \\ Relationship, Ethics, and Professionalism in the \\ Integrated Medical Curriculum
}

PALAVRAS CHAVES:

- Educação Médica;

- Mudança Curricular;

- Saúde da Família;

- Atenção Básica à Saúde.

\section{KEYWORD:}

- Medical Education;

- Curriculum Change;

- Family Health;

- Primary Health Care.

REVISTA BRASILEIRA DE EDUCAÇ̄̃o MÉDICA
Angélica Maria Bicudo Zeferino Maria de Lurdes Zanolli

Maria Ângela Reis de Góes Monteiro Antonio

\section{RESUMO}

Introdução: Visando atualizar suas práticas pedagógicas, atender as exigências da comunidade, da reestruturação do sistema de saúde e os avanços tecnológicos, a Faculdade de Ciências Médicas da Universidade Estadual de Campinas implementou uma grande reforma curricular para alunos ingressantes de 2001. Objetivo: Descrever uma experiência de ensino voltada à integração dos conhecimentos para atenção aos indivíduos nas diversas fases da vida, dentro da realidade de assistência primária à saúde, com ênfase no conhecimento, nas habilidades clínicas, na responsabilização e nas atitudes humanísticas e éticas. Métodos: No novo currículo, a integração intra, inter e transdisciplinar foi estruturada em módulos interdepartamentais, inserção progressiva das disciplinas clínicas, contato mais cedo e progressivo do aluno com a sistema de saúde, preservando módulos integradores horizontais e verticais. A iniciação da prática clínica em Centros de Saúde tem, no quarto ano, 432 horas destinada a atendimentos clínico-ambulatoriais de assistência à criança, à mulher, ao adulto e ao idoso num contexto de saúde da família. A supervisão é realizada por professores, médicos assistentes da Faculdade e tutores selecionados entre os profissionais da rede primária de saúde. O Programa Nacional de Reorientação da Formação Profissional em Saúde Pró-Saúde facilitou a inserção e a parceria do curso de medicina com as UBS. O conteúdo teórico é integrado em seminários ministrados em dois períodos semanais e avaliado por meio de provas teóricas (conhecimento cognitivo). As habilidades e competências nas atividades clínicas são avaliadas por meio de discussões teórico-práticas quinzenais ao longo do estágio, avaliações clínicas estruturadas de atendimentos à criança, mulher e adulto, além da composicão de portfólio com planilha de atendimentos totais, casos selecionados para revisão e auto-crítica de aprendizado. Resultados: O módulo foi avaliado na forma de fóruns semestrais de discussão, com participação de discentes, docentes, tutores e gestores. Os grupos foram unânimes em considerar plenamente atingidos os objetivos de responsabilização, vínculo e ética, e parcialmente atingida a integração dos conteúdos teórico-práticos e trabalho em equipe. Conclusão: O currículo integrado propiciou uma visão clínica abrangente da família. Permitiu que o estudante se responsabilizasse e criasse vínculo com o paciente, entendendo a resolutividade e demandas da atenção básica à saúde por meio de sua vivência.

\section{ABSTRACT}

Introduction: In order to update its teaching practices and meet the community's needs and restructuring of the health system and technological advancements, the School of Medical Sciences at the State University in Campinas implemented a major curricular reform for incoming students in 2001. Objective: Describe a teaching experience focused on the integration of knowledge for individual care in the various life phases, within the reality of primary healthcare, with an emphasis on knowledge, clinical skills, accountability, and humanistic and ethical attitudes. Methods: In the new curriculum, intra-, inter-, and cross-disciplinary integration was organized in inter-departmental modules, progressive participation in clinical disciplines, students' earlier and progressive contact with the health system, maintaining horizontally and vertically integrated modules. Initiation in clinical practice in Health Centers in the fourth year of school, with 432 hours dedicated to outpatient clinical care for children, women, adults, and the elderly in the context of family health. Supervision is provided by faculty, clinical staff from the medical school, and tutors selected from among the primary healthcare system staff. The National Program for Reorientation of Professional Training in Health (Pró-Saúde) facilitated participation and partnership between the medical school and the primary care units. Theoretical content is provided in seminars during two weekly periods, with grading based on written tests (cognitive knowledge). Skills and competencies in clinical activities are evaluated by means of theoretical and practical discussions every other week throughout the internship, structured clinical evaluations of care provided to children, women, and adults, in addition to the portfolio composition with the total number of consultations, selected cases for review, and self-criticism of the learning process. Results: The module was evaluated through discussion forums held once a semester with students, professors, tutors, and administrators. The groups agreed unanimously that the project had fully achieved the goals of accountability, bonds, and ethics, having partially achieved the integration between theoretical and practical course content and teamwork. Conclusion: The integrated curriculum fostered a comprehensive view of the family. It allowed students to take responsibility and create ties with patients, understanding the service's case-resolution capacity and demands of primary care through their direct experience.

'Universidade Estadual de Campinas, Campinas, SP, Brasil. 


\section{INTRODUÇÃO}

A Faculdade de Ciências Médicas (FCM) da Universidade Estadual de Campinas (Unicamp) iniciou, em 1998, um movimento de mudança objetivando reestruturar o currículo médico e definir um modelo pedagógico que contemplasse os desafios de formar médicos cidadãos. Esse movimento teve início com um grande seminário que envolveu a comunidade acadêmica, culminando com um consenso sobre a necessidade de mudança. As transformações requeridas exigiam, por um lado, estratégias de ensino que propiciassem despertar no estudante a capacidade de buscar os novos conhecimentos. Por outro, requeriam que os professores estivessem preparados e abertos para aprender a aprender e para promover a formação e a recuperação do profissional além do conhecimento técnico, com postura ética, humanística, capaz de resgatar a confiança e o respeito da comunidade, da família e, por fim, do seu cliente.

O modelo pedagógico que a cultura da escola permitia desenvolver diante dos desafios que sempre se apresentavam foi o de integração vertical e horizontal, buscando a inter, intra e transdisciplinaridade, com utilização de diferentes estratégias de ensino e cenários para a abordagem dos conteúdos, incorporando precocemente os casos clínicos como problematizadores do aprendizado.

A discussão para as mudanças curriculares na FCM vieram ao encontro das necessidades apontadas pelo Relatório da Comissão Interinstitucional Nacional de Avaliação do Ensino Médico (Cinaem), que buscava a melhoria do ensino. Entre as recomendações desse relatório incluíam-se: a diversificação dos cenários de prática; integração ensino-serviço de saúde do Sistema Único de Saúde (SUS); atuação prática precoce do estudante nas comunidades ou nos serviços (integração ensino-serviço-comunidade). Tais recomendações coincidiam com algumas das diretrizes do currículo proposto pela Comissão de Reforma Curricular, instituída como Comissão Supradepartamental Assessora da Diretoria, para ser a responsável pela implantação e pelo acompanhamento do novo currículo na FCM.

No modelo proposto, a integração horizontal e vertical proporcionou o desaparecimento das disciplinas de catálogo do curso, as quais foram substituídas por módulos compostos por diferentes áreas de conhecimento. Esse processo facilitou as mudanças necessárias e propiciou a descompartimentalização do aprender a cuidar, enxergando o ser humano mais completo, com a sua biologia, suas doenças, seu contexto de vida, seu ambiente e suas emoções.

Com a integração do básico com o clínico, o treinamento em serviço teve início real no quarto ano do curso, por meio da inserção do estudante na prática clínica e cirúrgica, fazendo diagnósticos e propondo tratamento das doenças mais prevalentes na comunidade. Para atingir o objetivo de formar um médico geral competente técnica, ética e humanisticamente, capaz de trabalhar em equipe, incorporar a tecnologia, ter espírito crítico e transformador em relação ao sistema de saúde, respeitando o contexto socioeconômico e a autonomia do paciente, houve a necessidade de inserir o estudante em um cenário real na base do sistema: na atenção primária.

Sob esse aspecto, há de se considerar também que a formação de recursos humanos tem necessidade de mudanças diante do contexto das reformas do setor da saúde. Considerando-se que a formação médica deve atender tanto as exigências da sociedade quanto a prestação dos serviços, a reforma na educação médica deve promover uma integração ensino-serviço. Esse é um grande desafio porque, para muitos professores, representa o afastamento das atividades científicas ou de treinamento de especialistas dentro dos muros da escola médica. Assim, a inserção precoce do estudante na comunidade, fazendo diagnósticos de necessidades e propondo intervenções de educação e saúde, realizadas no $1^{\circ}$ e $2^{\underline{o}}$ anos colaboram para aproximar o futuro médico e o professor da realidade social.

O módulo de Atenção Integral à Saúde, vivenciado durante o $4^{\circ}$ ano, contribui no vínculo do aluno com seu cliente, permite atender a família em contextos diferentes, criar responsabilização e aprender a trabalhar em equipe. Isso ocorre por meio do atendimento à saúde da criança e do adolescente, da mulher, do adulto e idoso, de forma integrada nas Unidades Básicas de Saúde.

\section{ESTRATÉGIAS}

A Reforma Curricular do Curso de Medicina da Faculdade de Ciências Médicas foi implantada no ano de 2001, passando de um currículo tradicional, estruturado em disciplinas sob responsabilidade individual dos departamentos, para um currículo integrado, horizontal e verticalmente estruturado em módulos gerenciados por docentes de vários departamentos que integram o básico clínico desde o primeiro ano do curso. A integração ensino-serviço e a ampliação dos cenários de prática começam no primeiro ano com o módulo Ações de Saúde Pública. Os estudantes são distribuídos em cinco grupos, compostos de dez a doze, acompanhados por um supervisor docente em dez Unidades Básicas de Saúde, onde realizam um trabalho de diagnóstico na comunidade. O objetivo aqui é a construção do conhecimento por intermédio de um trabalho didático que parte da experiência em campo e das vivências e concepções prévias dos alunos. Por meio do trabalho tutorado por docentes junto a um território de saúde de Campinas, as 
concepções prévias são problematizadas buscando um novo patamar de teorização. O módulo conta com encontros para apresentação dos trabalhos em campo, sem aulas teóricas.

No segundo ano do curso médico, o módulo Saúde e Sociedade possibilita ao aluno estabelecer um diálogo com a comunidade, buscando compreender os processos de adoecimento das pessoas e os diversos modos como elas são assistidas em seus problemas. O processo saúde-doença, a organização das práticas e as políticas públicas em relação à saúde são os eixos básicos para a análise da articulação do módulo. Os alunos são divididos em cinco grupos, e cada grupo desenvolve seu trabalho junto a um Distrito de Saúde (DS) do município. Os grupos são supervisionados diretamente por professores, auxiliares didáticos e profissionais de nível central, distrital e local da Secretaria de Saúde de Campinas (SMS). A escolha dos temas responde, ao mesmo tempo, à demanda da SMS e às necessidades de formação dos alunos, futuros médicos.

No terceiro ano, o módulo Epidemiologia e Saúde trabalha com os dados obtidos nos dois módulos anteriores, apresentando os principais conceitos de epidemiologia descritiva, com objetivo de permitir a compreensão do papel e da relevância da epidemiologia para a saúde pública e na atenção individual ao paciente.

No quarto ano, o módulo Atenção Integral à Saúde busca contribuir para a formação geral do médico, por meio da prática clínica em situações de atendimento primário em saúde da criança e do adolescente, da mulher, do adulto e do idoso. Esse módulo se destina ao desenvolvimento do raciocínio clínico, da compreensão do processo diagnóstico e terapêutico da prática da relação médico-paciente, da responsabilização e da vivência da atenção primária integral à saúde fora do ambiente hospitalar.

\section{Objetivos e Possibilidades}

Um dos principais objetivos do módulo é a inserção do aluno na rede básica do sistema de saúde, permitindo-lhe a percepção e análise crítica do sistema atual e da importância e possibilidade concreta de se realizar atenção resolutiva à saúde da população com qualidade nesse nível de atuação.

$\mathrm{Na}$ atenção individual, são enfatizadas as doenças de maior prevalência. Embora constituam o campo fundamental de conhecimento do médico após sua inserção no mercado de trabalho, essas doenças têm sido pouco observadas na formação, tradicionalmente centrada no contexto hospitalar. As discussões são ampliadas para que o aluno desenvolva a compreensão da interação entre o social e o individual, recuperando os determinantes coletivos dos problemas individuais e as questões individuais dos problemas coletivos, bem como suas soluções.

O graduando tem a possibilidade de praticar anamnese e exame físico, registrar as informações de modo claro e ordenado e valorizar o que achar relevante para cada caso. As condutas diagnósticas e terapêuticas para as situações mais comuns, as prescrições, são realizadas sob supervisão, a partir da qual se enfatiza a importância da adequação da dieta e das doses de medicamentos à idade e à massa corporal do paciente, integrando os processos diagnóstico e terapêutico e propostas de seguimento para os casos que acompanhe.

As atividades de vigilância à saúde, como atendimento domiciliar, elaboração de projeto terapêutico, ações de Saúde Coletiva, Ocupacional e Ambiental, Patologia Clínica e Radiologia complementam o módulo.

\section{Cenários de Prática - Organização do Módulo}

As atividades de atendimento individual são exercidas na forma de consultas, as quais são complementadas por discussão de casos com tutores e professores, com outros membros da equipe de saúde e participação em reuniões de projeto terapêutico.

Os Centros de Saúde que são campo de estágio foram selecionados em parceria com a Secretaria Municipal de Saúde, por meio do Centro de Treinamento em Saúde (Cets). Foram consideradas as necessidades de espaço físico e a proximidade com a Universidade, pois no período da manhã os alunos têm estágio no campus, restando pouco tempo para o deslocamento até as Unidades Básicas de Saúde (UBS) para início das atividades do período da tarde.

Os alunos são divididos em 12 grupos de nove ou dez alunos cada um, sendo que vinte alunos (dois grupos) ficam fixos em uma UBS durante os dois semestres, sempre com os mesmos supervisores. Cada UBS recebe, três tardes por semana, dez alunos de um grupo fixo que se revezam com os outros dez a cada semana, por cerca de 19 semanas e com um período de quatro semanas de férias no meio dos dois semestres. Dessa maneira, os mesmos alunos estão no serviço a cada 15 dias, o que facilita programar retornos e manter o acompanhamento dos pacientes.

Cada tarde contempla duas áreas: às quartas-feiras, cinco alunos ficam na área de Pediatria e cinco alunos na área de Ginecologia; às quintas-feiras, as áreas são Pediatria e Clínica Médica; e, às sextas-feiras, as áreas são de Clínica Médica e Ginecologia.

Portanto, dentro do objetivo do módulo de atender a família, o mesmo aluno atende a criança e, durante a anamnese, descobre que a avó ali presente é hipertensa e está sem con- 
trole da doença. Naquele momento, já convida a avó a marcar consulta com ele mesmo no dia em que estará na clínica. $\mathrm{O}$ aluno é estimulado a valorizar o vínculo com o paciente atendendo às intercorrências de seus pacientes sempre que se apresentarem, mesmo que em dia não designado para a clínica correspondente. Por exemplo: a mãe que traz seu filho para puericultura e ainda não fez sua revisão puerperal; no dia seguinte, quando o mesmo aluno estará na ginecologia com o docente e tutor específico, ele já terá conhecido e atendido a família, responsabilizando-se pelo cuidado. O próprio aluno fica encarregado de fazer o processo integrativo.

\section{Supervisão dos Graduandos}

É papel do docente da FCM (em cada clínica ou especialidade) dar supervisão às atividades dos alunos e contribuir na discussão dos casos atendidos, cobrindo a UBS e as equipes de saúde da família correspondentes.

O trabalho conta com docentes pediatras, clínicos e ginecologistas. Cada UBS deve ter uma equipe de profissionais que se responsabilize por todas as atividades realizadas pelos alunos. As equipes são constituídas por um docente ou médico assistente da FCM e um profissional da PMC, o qual é denominado tutor.

Os tutores são profissionais que acompanham os atendimentos e discutem os casos dividindo a função com os docentes. Trabalham na Rede Municipal de Saúde e, após processo seletivo, utilizam quatro horas semanais de sua carga horária em atividades de supervisão dos alunos do módulo. Os tutores devem atuar como facilitadores do ensino extramuros, aproximando os docentes e alunos das rotinas e atividades da UBS, garantindo o cumprimento de normas e condutas já estabelecidas.

Os candidatos a tutores, com perfil para o ensino das áreas de Pediatria, Clínica, GO e Saúde Coletiva, foram selecionados em parceria com o Cets. Os selecionados fizeram uma capacitação, na qual eram sensibilizados para a docência e esclarecidos sobre o módulo, objetivos do ensino-aprendizagem e papel compartilhado entre rede de saúde e Universidade na formação de recursos humanos.

\section{Desafios}

Uma grande dificuldade que se impõe é o espaço físico, que apresenta condições restritas, como o número insuficiente de salas para o atendimento individual e de grupo dentro das seis UBSs. Sabe-se que a rede de saúde pública deve ser campo de estágio para a formação de profissionais para o SUS, porém não está preparada estruturalmente para receber alunos, uma vez que para o ensino de qualidade e com metodologias ativas, em pequenos grupos para discussão dos casos clínicos e participação em projetos terapêuticos, é necessário um mínimo de estrutura, o que não existe na grande maioria das UBSs.

Para minimizar esse problema, a faculdade investiu financeiramente na reforma de UBSs para propiciar ensino com melhor estrutura física. Acredita-se que os alunos devem vivenciar o Sistema Único de Saúde (SUS) com dignidade para o usuário e profissional trabalhador, e não um sistema carente que oferece cuidado de má qualidade e sem conforto. Assim, há de se compreender que salas com pias para lavar as mãos é uma necessidade básica e que o SUS não é medicina para pobre, e sim é o sistema de saúde para todos. Por isso, a ideia é iniciar este ano ainda a reforma e ampliação da terceira UBS para campo de estágio. Há de se destacar que o Pró-Saúde auxiliou e propiciou, juntamente com a Faculdade, a melhoria da estrutura física e de equipamentos de trabalho para as UBSs. A partir da formação da Comissão Gestora Local, estabeleceu uma interlocução com a rede básica que muito tem auxiliado no gerenciamento da parceria e também dos conflitos gerados pela dificuldade de estabelecer ensino nas UBSs.

Outro desafio é a dificuldade em fixar docentes e médicos-assistentes, na sua maioria especialistas, nessas práticas, à exceção das áreas de Pediatria e Saúde Coletiva, que utilizam esses campos de prática há mais de 30 anos. A Clínica Médica e a Obstetrícia e Ginecologia ainda têm dificuldades no ensino fora do ambiente hospitalar. O enfrentamento desse problema requer uma política de ensino mais moderna, que consiga entender a importância dos diversos cenários de prática na formação de um profissional mais completo e reconheça o mérito desses profissionais.

\section{Submódulos}

As atividades teórico-práticas do submódulo Saúde Coletiva também são realizadas nas mesmas UBSs em encontros com grupos de dez alunos supervisionados por docentes do Departamento de Medicina Preventiva e apoio dos tutores de Saúde Coletiva. Este submódulo tem como principais objetivos conhecer o trabalho em saúde e a gestão do cuidado na atenção básica, envolvendo as necessidades de saúde, as ações e tecnologias de cuidado, os fluxos da atenção, o trabalho em equipe, as responsabilidades, a multiprofissionalidade e o olhar dos usuários sobre as ações e o serviço, assim como analisar as informações disponíveis no Núcleo de Saúde Coletiva da unidade e ações de Vigilância da Saúde.

Outros submódulos de apoio diagnóstico complementam conhecimentos na atenção integral à saúde:

Patologia Clínica - objetiva fornecer subsídios teórico-práticos para indicação e interpretação dos exames laborato- 
riais relacionados às doenças de maior prevalência em todas as faixas etárias atendidas na rede básica do sistema de saúde.

Radiologia - objetiva fornecer subsídios teórico-práticos para indicação e interpretação dos exames radiológicos relacionados às doenças de maior prevalência em todas as faixas etárias atendidas na rede básica do sistema de saúde.

Saúde Ambiental - capacita o aluno em Toxicologia Clínica e Epidemiológica com noções de toxicologia aplicáveis na atenção ao indivíduo e à coletividade.

Saúde Ocupacional - ressalta, na formação geral do médico, a importância do trabalho em si, seu ambiente e as condições em que se realiza, além de apresentar as doenças relacionadas ao trabalho, com enfoque nas situações mais prevalentes no nível primário de atenção à saúde.

O módulo Atenção Integral à Saúde tem um submódulo que consiste em um curso teórico das condições clínicas mais prevalentes, com abordagem integrada das áreas da criança, adolescente, mulher, adulto e idoso.

\section{AVALIAÇÃO DISCENTE NAS ATIVIDADES DAS UBSS:}

Cada submódulo tem seu sistema de avaliação, que é computado na média final do módulo. O aluno é aprovado se obtiver média final maior ou igual a sete; caso contrário, realiza um Exame Final para avaliação cognitiva e discussão de casos clínicos.

O módulo Atenção Integral à Saúde no submódulo UBS experimentou uma avaliação formativa e somativa, que se mostrou muito interessante, envolvendo grande esforço dos docentes e tutores. Foi utilizado o "portfólio" individual, no qual foram incluídas as planilhas de atendimento diário com diagnósticos, a avaliação de relatórios orientados sobre casos de Clínica, Pediatria e Ginecologia e revisão bibliográfica sobre um tema que se considerou relevante em cada caso discutido. $\mathrm{O}$ intuito aqui foi fazer o aluno refletir sobre seu atendimento ambulatorial, sobre suas dificuldades e facilidades, sobre suas carências no estágio. No início do segundo semestre, a correção dos "portfólios" foi discutida em cada UBS pelos docentes e tutores, buscando orientar os alunos sobre as carências observadas e colher suas sugestões. No segundo semestre, após a fase piloto, também se agregou a Avaliação Estruturada do Atendimento com formulários por clínica.

O módulo foi avaliado no final do primeiro semestre em uma Oficina de Avaliação com todos os parceiros envolvidos, dois representantes dos alunos de cada UBS, docentes, tutores e gestores (da UBS e da SMC). A estratégia utilizada foi uma introdução com retomada dos objetivos da integração e formação dos profissionais voltados para a realidade vivida sob a responsabilidade da coordenação do módulo e representantes das SS, seguida por divisão em seis grupos (UBS). Cada grupo discutiu os itens: responsabilização, vínculo médico-paciente, ética, integração de conteúdos teórico-práticos e trabalho em equipe. $\mathrm{O}$ resultado foi considerado positivo para todos os itens e confirmou que o currículo integrado pode propiciar atenção integral à saúde dos indivíduos e ensino médico dentro da rede primária, melhorando a integralidade das ações e, por consequência, o aprendizado do "ser médico". No segundo semestre, processo similar considerou a experiência exitosa da mesma forma.

\section{CONCLUSÕES}

A integração ensino-serviço atingiu seu ponto alto no quarto ano com o módulo Atenção Integral à Saúde, quando o aluno agrega ao conhecimento extramuros do primeiro e segundo anos a vivência como médico de uma mesma UBS, com a mesma equipe de saúde, os mesmos professores e colegas durante todo o ano letivo. Esse modelo possibilitou a responsabilização, o vínculo com o usuário e a equipe de profissionais, o exercício da ética com o usuário e com os colegas, associando teoria e prática, entendendo a dinâmica e a importância do atendimento da família dentro do Programa de Saúde da Família. O Pró-Saúde cumpriu o seu objetivo principal de facilitar a formação de profissionais com inserção na atenção básica durante a graduação.

Com a formação de profissionais médicos cidadãos direcionados para a saúde da maioria da população com responsabilidade, vínculo e compromisso médico-paciente, sabendo trabalhar e interagir com a equipe de saúde e com a família, com ética e com excelente conhecimento técnico-científico. Desse modo, está-se formando um profissional humanizado, voltado para as necessidades da população, hábil para atuar em todos os níveis de atenção à saúde.

\section{REFERÊNCIAS CONSULTADAS}

1. Comissão Interinstitucional Nacional de Avaliação das Escolas Médicas. Relatório geral 1991-1997: avaliação do ensino médico no Brasil. Brasília: CINAEM, 1997.

2. Almeida MJ. Educação e Saúde. Possibilidades de Mudança. Londrina: Ed. UEL; 1999.

3. Fraga CF. Ensino Médico: bases e diretrizes para sua reformulação. Rev Bras Educ Med. 1986;10 (2).

4. Souza MPG, Rangel M. Avaliação: um Impasse na Educação Médica. Rev Bras Educ Med. 2003;27(3):213-22.

5. Schneider CD, Meek PM, Bell IR. Development and validation of IMAQ: Integrative Medicine Attitude Questionnaire. BMC Medical Education. 2003; 3: 5. 
6. Solomon DJ, Laird-Fick HS, Wkeefe C, Noel MM, Noel T. Using a formative simulated patient exercise for curriculum evaluation. BMC Medical Education. 2004; 4:8.

7. Ronzani TM, Ribeiro MS. Identidade e Formação Profissional dos Médicos. Rev Bras Educ Med. 2003;27(3): 229-36.

8. Amaral E, Zeferino AMB, Nadruz Junior W, Antonio MARG, Sarian LOZ, Inhaia C, Leite RC, Mennin S. Successful accomplishment of educacional goals with clinical experience at public primary care facilities, 01/2007. Med Teach.2007;29:600-5.

9. Domingues RCL, Amaral E, Zeferino AMB. Global overall rating for assessing clinical competence: what does it really show?, 09/2009. BMC Medical Education. 2009;43(9):883-6.

10. Domingues RCL, Amaral E, Zeferino AMB. The different perspectives in the assessment of learners during supervised clinical clerkship, 08/2009. Rev Assoc Med Bras. 2009; 55(4):458-62.

11. Souza PA, Zeferino AMB. Changes in medicine course curricula in Brazil encouraged by the Program for the Promotion of Medical School Curricula (PROMED), 01/2008. BMC Medical Education. 2008; 8:54.
12. Zeferino AMB. Os desafios das mudanças no currículo e na avaliação no curso de medicina. Campinas; 2008. Livre Docência - Universidade Estadual de Campinas.

\section{CONTRIBUIÇÃO DOS AUTORES}

Os três autores tiveram participação na elaboração intelectual e prática do relato de experiência no curso de medicina com o Pró-saúde.

\section{CONFLITOS DE INTERESSE}

Declarou não haver.

\section{ENDEREÇO PARA CORRESPONDÊNCIA}

Angélica Maria Bicudo Zeferino

Rua Madre Paulina, 66

Residencial Parque Rio das Pedras, Barão Geraldo - Campinas CEP. 13085-150 SP

E-mail: ambz@fcm.unicamp.br 